\title{
Radiological progression of hip osteoarthritis: definition, risk factors and correlations with clinical status
}

\author{
Maxime Dougados, Alice Gueguen, Minh Nguyen, Laurent Berdah, Michel Lequesne, \\ Bernard Mazieres, Eric Vignon
}

Clinique de

Rhumatologie,

Hôpital Cochin,

27 rue du Fbg

St-Jacques, 75014

Paris, France

$M$ Dougados

$M$ Nguyen

Unité 88 INSERM,

Hôpital National de

Saint Maurice,

14 avenue du Val

d'Osne, 94410 Saint

Maurice, France

A Gueguen

Laboratoires Negma, Immeuble Strasbourg,

Avenue de l'Europe,

78117 Toussus le Noble,

France

L Berdah

Hôpital Léopold

Bellan, 19 rue

Vercingétorix,

75014 Paris, France

M Lequesne

Service de

Rhumatologie,

CHU Rangueil,

31054 Toulouse Cedex,

France

B Mazieres

Service de

Rhumatologie

Pavillon F, Hôpital

Edouard Herriot,

69437 Lyon Cedex 03,

France

E Vignon

Correspondence to:

Maxime Dougados.

Accepted for publication

6 February 1996

\begin{abstract}
Objectives-To determine a cut off value for changes in radiological joint space width that allowed definition of radiological progression of hip osteoarthritis not related to measurement method errors and, thereafter, to determine factors predictive of radiological progression of hip osteoarthritis and to evaluate the correlations between clinical and radiological parameters.
\end{abstract}

Methods-A prospective, longitudinal (one year duration), multicentre study was made of patients with osteoarthritis of the hip (American College of Rheumatology criteria). Data on clinical activity (pain, functional impairment), demographic data (age, gender, body mass index), and femoral head migration (superolateral, superomedial, concentric) were collected when the patient entered the study; radiological grade (joint space width in millimetres at the narrowest point using a $0 \cdot 1$ mm graduated magnifying glass, evaluated by a single observer unaware of the chronology of the films) was recorded at the patient's entry to the study and after one year.

Results-Analysis of the means of the differences between two analyses performed by a single observer of 30 pairs of radiographs (one performed after an interval of one year) $(0.06(S D$ 0.23)) suggested that a change of more than $0.56 \mathrm{~mm}$ (2 SD) after a one year follow up could define progression of osteoarthritis of the hip. Of the 508 patients recruited, $461(91 \%)$ completed the one year follow up and radiological progression was observed in 102 $(22 \%)$. The factors predictive of radiological progression that were identified in the multivariate analysis were: radiological joint space width at entry $\leq 2 \mathrm{~mm}$, superolateral migration of the femoral head, female gender, Lequesne's functional index $>10$, age at entry $>65$ years (odds ratios $2 \cdot 11,4 \cdot 25,2 \cdot 51,2 \cdot 66,1 \cdot 90$, respectively). The level of clinical parameters (pain, functional impairment) and the amount of symptomatic treatment required (non-steroidal antiinflammatory drugs and analgesic intake) accounted for $20 \%(p<0.0001)$ of the variability of the changes in radiological joint space width over the one year study period.

Conclusion-These data suggest that radiological progression of hip osteoarthritis could be defined by a change in joint space width of at least $0.6 \mathrm{~mm}$ after a one year follow up period, is correlated with the changes in clinical status of the patients, and is related not only to demographic data (age, gender), but also to some specific characteristics of osteoarthritis (localisation, radiological severity, clinical activity).

(Ann Rheum Dis 1996; 55: 356-362)

The prevalence of hip osteoarthritis ranges from 7 to $25 \%$ in adults aged 55 years and older in the white European population. ${ }^{1-4} \mathrm{Hip}$ osteoarthritis is one of the main causes of disability among the elderly. The natural history of hip osteoarthritis has been poorly evaluated in the past, but available studies clearly demonstrated heterogeneity of disease progression, pointing out that in some patients the disease does not progress even after a long follow up period. ${ }^{5-8}$ In addition, there is not universal agreement as to the definition of progression of hip osteoarthritis: some studies define progression with reference to clinical parameters (functional disability, painful condition), ${ }^{679}$ others refer to the need for surgery, ${ }^{568}$ but most define progression by radiological parameters. ${ }^{689}$

Radiological changes combine signs related to bone changes (cysts, sclerosis, osteophytes) and signs probably related to cartilage loss (joint space narrowing). ${ }^{1011}$ The evaluation of joint space width by measuring either the interbone distance at the narrowest point ${ }^{12}{ }^{13}$ or the joint space area ${ }^{13-15}$ is considered the most sensitive technique. However, this evaluation results in a continuous variable - changes (in millimetres) in the joint space width at the narrowest point-and does not permit distinction between the patients whose disease shows progress anatomically and those in whom it does not. In a recent longitudinal study of knee osteoarthritis, a $2 \mathrm{~mm}$ change was chosen arbitrarily ${ }^{16}$ as the cut off above which a change in the joint space width could be considered to 
represent relevant progression of this anatomical parameter.

Whatever the definition of progression of hip osteoarthritis, it must combine sensitivity and clinical relevance. For instance, in the case of radiological definition, correlations between the changes in the clinical status of the patient and the radiological parameters must be evaluated.

Variables predicting the outcome of hip osteoarthritis are not well known. Some studies suggest that variables such as gender, ${ }^{17} 18$ age $^{58}$ and radiological patterns $s^{5817}$ might influence disease progression. Identification of such variables predicting the outcome of osteoarthritis is desirable in order to distinguish subjects having a high or low risk of developing severe disease.

Given the above factors, it seemed of interest to conduct a prospective longitudinal (one year) study in a large cohort of patients with painful hip osteoarthritis in order to determine the percentage of patients with radiological progression and the factors predisposing to this defined radiological progression, and to evaluate correlations existing between the clinical and radiological parameters.

\section{Patients and methods}

STUDY DESIGN

This was a multicentre, prospective, longitudinal, three year study approved by the Ethics review board of Cochin Hospital (Paris), aimed at evaluating in a double blind placebo controlled trial the effect of diacerhein on the radiological progression of hip osteoarthritis (ECHODIAH study). The results presented here are those obtained at the end of the first year of follow up of all the recruited patients without breaking the randomisation code of the continuing treatment trial.

\section{PATIENTS}

After written informed consent was obtained, patients fulfilling the American College of Rheumatology (ACR) criteria for the diagnosis of hip osteoarthritis were recruited for the study. Other criteria of eligibility included: age between 50 and 75 years, painful condition defined by the presence of daily pain during at least one month in the past three; absence of secondary hip osteoarthritis defined by presence or past history of hip fracture, inflammatory rheumatic disease, osteonecrosis, Paget's disease, etc; a joint space width $>1 \mathrm{~mm}$ at the narrowest point; absence of medial or axial femoral head migration, or both, on radiographs (fig 1).

\section{DATA COLLECTION}

The following characteristics were noted at baseline: demographic data including age, gender, body mass index, history of hip osteoarthritis including date of first symptoms, and date of diagnosis; characteristics of osteoarthritis including the radiographic pattern of migration of the femoral head, classified as

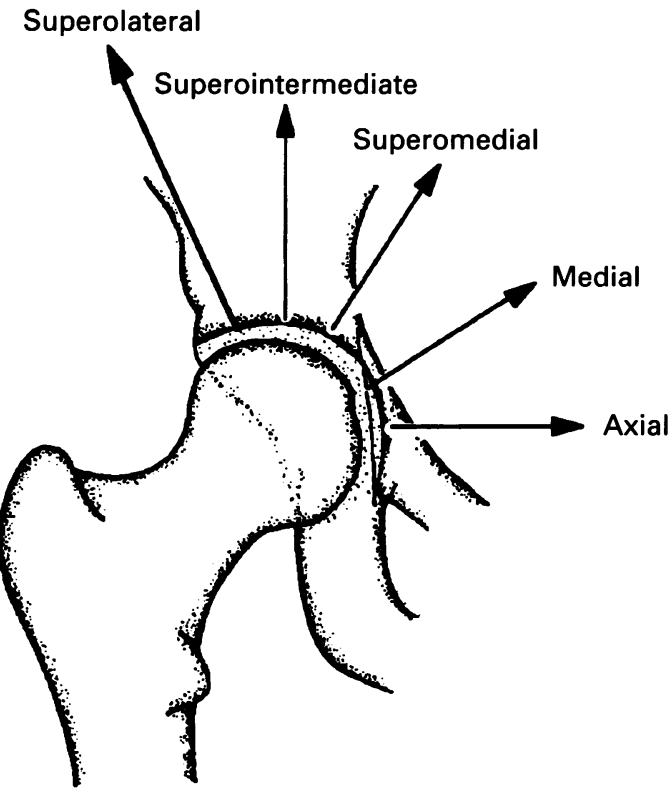

Figure 1 Different radiographic patterns of osteoarthritis of the hip. Medial and axial femoral head migration were criteria for exclusion from the study.

superolateral or superointermediate, superomedial or concentric (according to Ledingham et $a l^{5}$ ) (fig 1), and severity (Kellgren and Lawrence grading system); bilaterality (ACR criteria); the presence of polyarticular involvement evaluated using the articular score derived from the Landsbury index as reported previously. ${ }^{19}$

Each patient was evaluated by a single observer at entry and after one, three, six, nine, and 12 months. During the study, the patients were asked to maintain the doses of drugs taken to treat their symptoms (non-steroidal anti-inflammatory drugs (NSAIDs) or analgesics) at the minimum level. In addition, before each visit, the patients were asked to observe a washout period (seven days for NSAIDs and three days for an analgesic).

At each visit, the following data were collected: pain occurring during physical activities during the previous two days (100 mm visual analogue scale (VAS)); percentage of painful days during the month preceding the visit; overall assessment of disease activity during the previous two days (100 mm VAS); functional disability evaluated by the Lequesne index (a questionnaire on daily activities during the previous seven days; score 0 to $24^{20}$ ); mobility impairment (evaluated by the intercondylar distance).

The amount of rescue treatment between two visits was also recorded. Because patients might take different drugs we used both an equivalent NSAID score ${ }^{21}$ that made it possible to calculate a mean daily NSAID score (for example, a patient taking $100 \mathrm{mg}$ indomethacin daily has a score of 10 , which is the same if $\mathrm{s} / \mathrm{he}$ takes $150 \mathrm{mg}$ diclofenac or $20 \mathrm{mg}$ piroxicam), and an equivalent score of analgesics defined by the number of mean daily tablets taken by the patient and considering one tablet of paracetamol to be equivalent to one tablet of dextropropoxyphene. 
In addition, we recorded at each visit the percentage of days since the previous visit during which the patient had to take one of these drugs.

RADIOLOGICAL EVALUATION

At entry and after one year, a weight bearing anteroposterior pelvic radiograph was performed. All the films were collected and analysed by a single observer (MN). The pairs of films were blinded for patient identity and date. A randomisation list was used to blind the chronology-each film for one patient was labelled A or B.

For the analysis, the two films of a single patient ( $A$ and $B$ ) were placed side by side on a light box and the narrowest point (identical for the two films) was selected by the investigator. The interbone distance was measured using a $0.1 \mathrm{~mm}$ graduated magnifying glass.

To evaluate intra- and interobserver reliability (see below), 30 pairs of films representative of the spectrum of the radiological severity of the disease were selected.

STATISTICAL ANALYSIS

Definition of radiological progression-In order to determine a cut off value of changes in joint space width that allowed definition of radiological progression of hip osteoarthritis not related to measurement method errors, we used the method proposed by Bland and Altman. ${ }^{22}$ This method involves calculating the mean of the differences between two analyses, and can be used to evaluate interand intraobserver reliability. For this purpose, we selected 30 radiographs representative of the spectrum of the disease in terms of severity and of patterns of femoral head migration. The differences between two observers (MD and $\mathrm{MN}$ ) were used to evaluate interobserver reliability, and those between two analyses performed by the same observer $(\mathrm{MN})$ with an interval of one month were used to evaluate intraobserver reliability. The analysis was performed not only on the 30 films taken at entry, but also on the 30 pairs of films taken at entry and after one year. This methodology enabled us to evaluate the reliability not only of the absolute value of the joint space width, but also of the changes in joint space width. In addition, we evaluated the intraclass coefficient of correlations existing between the observers (interobserver reliability) and between two analyses (intraobserver reliability), for both the absolute and the relative value (changes in joint space after one year of follow up).

Factors predictive of radiological progression of osteoarthritis of the hip-Multivariate analyses were performed to identify factors predictive of radiological progression of osteoarthritis of the hip. In these analyses, independent variables were collected at entry: demographic data, characteristics of osteoarthritis, clinical activity, and radiological severity. Changes in the radiological joint space width defined the dependent variable (the continuous variable (change in $\mathrm{mm}$ )) in the multiple linear regression and the dichotomous variable (radiological progression: yes/no) in the multiple logistic regression.

Correlations between clinical status and radiological parameters-For this purpose, multivariate analyses were performed in which the radiological parameter defined the dependent variable and the clinical (pain, functional disability) and therapeutic variables (daily NSAID and analgesic score, percentage of days taking NSAIDs, analgesics, or both) defined the independent variables. A multiple linear regression was performed at entry to evaluate correlations between the absolute value of joint space width and clinical parameters, but also after one year of the study to evaluate correlations between the changes of joint space width and the changes (area under the curve) in clinical and therapeutic parameters. In this longitudinal study, a multiple logistic regression was also performed in which the independent variables were similar to those of the multiple linear regression, but in which the dependent variable was the dichotomous variable (radiological progression: yes/no). Because data concerning the clinical and therapeutic parameters were missing for some patients for various visits of the study, two analyses were conducted: one in the group of patients without any missing data (completer group) and one in the whole group of patients. For this latter analysis, the technique of LOCF (Last Observation Carried Forward) was applied.

\section{Results}

PATIENTS AND STUDY COURSE

Table 1 summarises the main characteristics of the 508 patients recruited to the study. All these patients except one were enrolled in the clinical trial. The last patient screened at entry completed the one year follow up period and was therefore kept in this epidemiological study. During the one year follow up of the study, 32 patients underwent hip arthroplasty after a mean of eight months of follow up (range 3-12 months). Among these patients, a pelvic radiograph obtained before their

Table 1 Baseline characteristics of the 508 patients with hip osteoarthritis

\begin{tabular}{lc}
\hline Parameter & Results \\
\hline Demographic data & \\
Age (years) & $63(7)$ \\
Sex (female/male) & $303 / 205$ \\
Body mass index & $26(4)$ \\
Hip osteoarthritis & \\
Disease duration (years) & $4 \cdot 6(4 \cdot 8)$ \\
Femoral head migration & \\
$\quad$ Superolateral & $298(59 \%)$ \\
Superomedial & $160(31 \%)$ \\
Concentric & $50(10 \%)$ \\
Clinical activity & $45(20)$ \\
Pain (VAS) & $7 \cdot 8(2 \cdot 6)$ \\
Lequesne's index & \\
Radiological severity & $14(3 \%)$ \\
Kellgren \& Lawrence scoring system & $356(70 \%)$ \\
I & $136(27 \%)$ \\
II & $2(<1 \%)$ \\
III & \\
IV &
\end{tabular}

Values are means (SD) or number (\%). 
Table 2 Intra-and interobserver reliability of measurement of joint space width based on the analysis of the mean of the differences (Bland and Altman technique ${ }^{22}$ )

\begin{tabular}{lll}
\hline Analysis & \multicolumn{2}{l}{ Mean of differences } \\
\cline { 2 - 3 } & Intraobserver & Interobserver \\
\hline $\begin{array}{l}\text { Joint space width (one single } \\
\text { radiograph) }\end{array}$ & $0.02(0.40)$ & $0.07(0.52)$ \\
$\begin{array}{l}\text { Changes in joint space width } \\
\text { (two radiographs, one after a } \\
\text { one year interval) }\end{array}$ & $0.01(0.28)$ & $0.10(0.34)$ \\
\hline Values are mean (SD). & & \\
\end{tabular}

operation was available in 23 , and was taken into account in the analysis. Among the other 476 patients, a one year follow up including a radiological evaluation was completed in 438. Therefore, after one year, a radiological evaluation was available in 461 patients (91\%).

\section{RADIOLOGICAL PROGRESSION OF HIP OSTEOARTHRITIS}

Table 2 summarises the results for intra- and interobserver reliability based on analysis of the mean of the differences. Differences in evaluation of changes in joint space width (analysis of two radiographs, one performed after a one year interval) between two analyses performed by the same observer were 0.01 (SD 0.28$) \mathrm{mm}$. For this study, radiological progression was therefore defined by a change in joint space width greater than the $95 \%$ confidence interval of these differences-that is, a change of at least $0.6 \mathrm{~mm}$. The intraclass coefficient of correlation evaluated for the intra- and interobserver reliability was 0.85 and 0.78 , respectively, when calculated on one single radiograph per patient and was 0.77 and 0.68 , respectively, when calculated on the changes in joint space width after one year of follow up.

In the 461 patients evaluated, joint space width changed from $2 \cdot 37(0.80) \mathrm{mm}$ at entry to $2.04(0.98) \mathrm{mm}$ after one year-that is by $0.33(0.53) \mathrm{mm}(\mathrm{p}<0.0001)$. In the 23 patients for whom a radiograph taken before surgery was available, joint space width changed from $2.01(0.83) \mathrm{mm}$ at entry to 1.03 $(1.08) \mathrm{mm}$ just before surgery-a change of $0.97(0.73) \mathrm{mm}(\mathrm{p}<0.0001)$. Figure 2 summarises the individual results: radiological

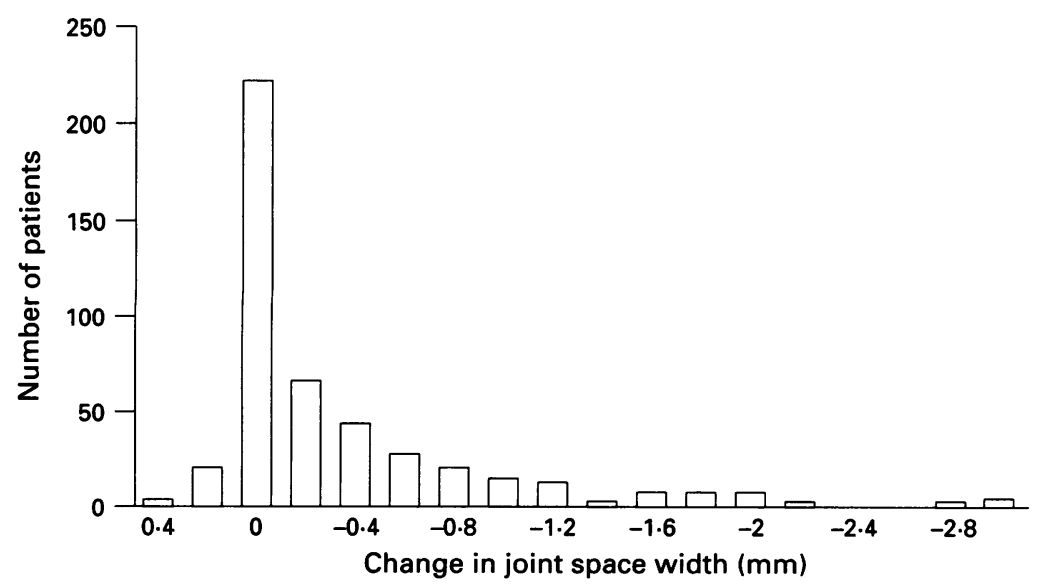

Figure 2 Distribution of changes in radiological joint space width at the narrowest point in 461 patients with osteoarthritis of the hip, after one year of follow up. progression was observed in 102 patients $(22 \%)$, but radiological improvement was never observed. Radiological progression was observed in 16 of the 23 patients who underwent hip arthroplasty (70\%).

FACTORS PREDICTIVE OF RADIOLOGICAL PROGRESSION OF OSTEOARTHRITIS OF THE HIP The multiple linear regression in which the dependent variable was defined by the changes in joint space width (in $\mathrm{mm}$ ) and the independent variables were defined by the clinical and radiological parameters collected at entry was conducted in 458 patients and enabled the identification of eight predictive variables: age at entry greater than 65 years; female gender; superolateral migration of femoral head; ulilateral hip osteoarthritis; Kellgren and Lawrence radiological score greater than 3; Lequesne functional index greater than 10 ; more than $90 \%$ of painful days during the month preceding entry visit; greater mobility (intercondylar distance greater than over $40 \mathrm{~mm}$ ). All these variables explained $15 \%$ of the variability of the changes in radiological joint space width $(p<0.0001)$.

The multiple logistic regression analysis in which the dependent variable was defined by the presence or absence of radiological progression and the independent variables were defined by the clinical and radiological parameters collected at entry was also conducted in the 458 patients and enabled identification of five predictive variables (table 3 ) that included demographic data (age, gender, characteristics of osteoarthritis (localisation of femoral head migration and radiological severity), and parameters evaluating clinical activity (Lequesne's functional index). In order to evaluate the consistency of the results obtained, we performed a multivariate analysis in which two different thresholds were adopted for rate of change- the first defined by the 10th centile (a change of at least $0.46 \mathrm{~mm}$ ), and the second defined by the 20th centile (a change of at least $0 \cdot 36$ ). Similar results were obtained (data not shown), strengthening the data obtained on determinants of progression when the original definition of radiological progression, a change of at least $0.6 \mathrm{~mm}$, was used.

Analyses of the correlations between age at onset of disease and the risk of radiological progression showed a radiological progression in $15 \%, 15 \%, 20 \%, 27 \%$, and $33 \%$ of patients aged less than 55 years $(n=79), 56-60$ years $(n=87), 60-65$ years $(n=110), 65-70$ years $(n=109)$, and more than 70 years $(n=73)$ at entry, respectively.

Table 3 Factors predictive of radiological progression of osteoarthritis of the hip

\begin{tabular}{lll}
\hline Parameter & Odds ratio & $95 \%$ confidence interval \\
\hline $\begin{array}{l}\text { Joint space width at entry } \\
\text { <2 mm }\end{array}$ & 2.11 & 1.30 to 3.44 \\
$\begin{array}{l}\text { Superolateral migration of } \\
\text { the femoral head }\end{array}$ & 4.25 & 2.26 to 8.01 \\
$\begin{array}{l}\text { Female gender } \\
\text { Lequesne's index >10 }\end{array}$ & 2.51 & 1.49 to 4.23 \\
Age at entry $>65$ years & 1.96 & 1.46 to 4.83 \\
\hline
\end{tabular}


Radiological progression was more frequently observed in patients with a joint space width less than $2 \mathrm{~mm}$ : frequencies were $29 \%, 30 \%, 16 \%, 18 \%, 19 \%$, and $21 \%$ among patients with a joint space width at entry less than $1.5 \mathrm{~mm}(\mathrm{n}=79), 1.5-2 \mathrm{~mm}(\mathrm{n}=87)$, $2-2.5 \mathrm{~mm}(\mathrm{n}=102), 2 \cdot 5-3 \mathrm{~mm}(\mathrm{n}=108)$, 3-3.5 mm $(\mathrm{n}=53)$, and more than $3.5 \mathrm{~mm}$ $(n=29)$, respectively.

Radiological progression was observed in $12 \%, 23 \%, 19 \%, 33 \%$, and $53 \%$ of patients with a Lequesne's functional index at entry less than $5(n=89)$, between 5 and $7 \cdot 5(n=145)$, between 7.5 and $10(n=152)$, between 10 and $12.5(n=57)$, and greater than $12.5(n=15)$, respectively.

Two additional parameters were recognised as potential predictive factors of radiological progression: gender and localisation. Radiological progression was observed in $26 \%$ of 272 women compared with only in $15 \%$ of 186 men, and in $25 \%$ of 292 patients with a superolateral or superointermediate femoral head migration compared with $11 \%$ of 135 patients with a superomedial femoral head migration. In the subgroup of 31 patients with a concentric pattern of joint space narrowing, radiological progression was observed in $35 \%$.

CORRELATIONS BETWEEN CLINICAL STATUS AND RADIOLOGICAL PARAMETERS

The multiple linear progression performed at entry of the patient to the study showed that all the clinical parameters (pain, functional disability) explained only $0.4 \%(p=0.44)$ of the variability of the radiological joint space width $(n=508)$. In contrast, the level of the clinical parameters and the amount of symptomatic treatment during the one year follow up period explained $20 \%(\mathrm{p}<0.0001)$ of the variability of the changes in radiological joint space width. This result was obtained with analysis both in the completer group (369 patients without any missing data: $r^{2}=20 \%$ ) and in the whole group of patients (460 patients: $r^{2}=20 \%$ ).

In order to evaluate the relationships between therapeutic and radiological parameters, we conducted multivariate analysis by including in a first step all the clinical parameters (pain, Lequesne's index, overall assessment), and then adding the therapeutic parameters (consumption score and the percentage of days of analgesic and NSAID treatment). After adjustment for the two clinical variables, the correlations between analgesic consumption and the changes in radiological parameter did not reach statistical significance $(p=0 \cdot 14)$, but there remained a statistically significant correlation between the NSAID consumption score and the changes in radiological parameter $(p<0 \cdot 01)$. After adjustment for both clinical variables and NSAID consumption, there was no longer a statistically significant correlation between the analgesic consumption score and the changes in radiological parameter $(p=0.49)$; however, a statistically significant correlation remained when the statistical model of the percentage of days taking analgesics was added $(p=0.014)$. After adjustment for the two clinical variables and for the analgesic consumption score, there remained a statistically significant correlation between the NSAID consumption score and changes in the radiological parameter $(p=0.0001)$. This statistically significant difference was retained when the percentage of days taking NSAIDs was added $(p=0.0004)$.

Similar results were obtained when a multiple logistic regression was conducted in which the dependent variable was defined by the presence or absence of radiological progression and the independent variables were defined by the clinical and therapeutic parameters collected during the one year of the study (data not shown).

\section{Discussion}

The findings of this study permit a definition of the degree of radiological progression that can be regarded as significant at the hip, given the reproducibility of joint space width measurement. It also suggests that $20 \%$ of patients progress radiologically after one year and that this progression is related not only to demographic data (age, gender), but also to some specific characteristics of osteoarthritis such as its clinical activity. Finally, the study also suggests that a statistically significant correlation exists between clinical and radiological parameters evaluating hip osteoarthritis; however, a longitudinal study design is required to demonstrate such a correlation. Analysis of these correlations also raises the question of the deleterious effect on cartilage resulting from NSAID intake in osteoarthritis.

Because of the characteristics of the study population and because of the study design (first part of a therapeutic trial), it is not possible to extrapolate the results of this study to a general population of patients with hip osteoarthritis. This study was focused on a particular subgroup of patients all of whom, for example, had a painful active disease and a particular pattern of osteoarthritis. These criteria might influence the results both in terms of radiological progression and in terms of potential predisposing factors of radiological progression. However, such characteristics are those commonly observed in daily practice and therefore, though it is not possible to extrapolate the results obtained to a general population of patients with hip osteoarthritis, they may be of interest in relation to other patients consulting rheumatologists because of a clinically painful condition.

The results presented here were obtained during the course of a continuing clinical trial evaluating the effects of a drug (diacerhein) that could interfere with the results related to the predisposing factors of progression. However, even in the event that a specific treatment effect occurred, it may be assumed that it was the same regardless of other variables such as age and gender. The lack of knowledge of the treatment group to which each patient belonged thus probably does not alter the conclusion of this study in terms of pre- 
disposing factors of radiological progression. Another outcome of the lack (in this part of the overall study) of evaluation of the potential effect of the treatment under investigation (diacerhein) is that the percentage of patients with radiological progression might be underestimated in this analysis. However, this potential underestimation lends further support of one of the main results of this study that suggests that radiological progression of hip osteoarthritis is easily detectable within a one year period of follow up.

There is no consensus concerning the definition of radiological progression. Methods assessing the severity of osteoarthritis using categorical variables, such as the Kellgren and Lawrence scoring system or the size of osteophytes, permit definition of progression by a change of at least one grade during the study period. However, the assessment of joint space width using a continuous variable such as measurement of the interbone distance at the narrowest point evaluated in millimetres (as in the present study) is currently accepted as the most sensitive technique. Evaluation of this specific radiological parameter seems to be related to patient positioning, at least in the subgroup of those patients with osteoarthritis who have a joint space width less than $2.5 \mathrm{~mm}$, as a mean decrease of $13 \%$ was observed when radiographs were taken with the patient in the standing position compared with the supine position. ${ }^{23}$ For this reason, in this study all radiographs were taken with the patient in a weight bearing position. In contrast, no particular guideline exists concerning the radiological procedure or other characteristics of patient positioning, though results of one study suggested that variations in the rotation of lower limbs or in the direction of the radiological beam, or both, did not influence the evaluation of joint space width. ${ }^{24}$ The presence of hip flexion deformities was not evaluated in the present study and could interfere with the measurement of joint space width; however, such a condition was probably uncommon in the patients recruited, as we excluded patients with advanced disease. In order to present the results as a dichotomous variable (progression yes/no), it was necessary to establish a cut off. An arbitrary value has been proposed for the evaluation of knee osteoarthritis (a change greater than $2 \mathrm{~mm}^{16}$ ), while, for the hip, Lequesne considered that a change of at least $0.5 \mathrm{~mm}$ might be clinically relevant. ${ }^{12}$ We now propose an objective definition of radiological progression based on analysis of its reproducibility (a change greater than $0.6 \mathrm{~mm}$ ) that, interestingly, is very close to that proposed by Lequesne (a change over $0.5 \mathrm{~mm}) .{ }^{10}$ Independent studies performed by other observers are required to confirm or refute the validity of this cut off value.

Using our proposed definition, and notwithstanding any treatment effects of the trial drug, we observed a radiological progression in $20 \%$ of the patients studied. This value both confirms the findings of previous studies indicating that some patients do not show radiological progression and suggests that it is possible to demonstrate a statistically highly significant deterioration in joint space width within one year follow up. A period of at least several years is usually considered necessary to observe such progression. Our results were undoubtedly biased by the characteristics of the patients studied: all had active disease and were older than 50 years at entry to the study-two parameters that can be considered potential risk factors for radiological progression. ${ }^{2}$ In addition, we excluded from this study patients with medial migration of the femoral head, and such a pattern has been reported to be correlated with slow progression of the disease. It is possible to argue, therefore, that different results could be obtained in other subsets of patients-for example, a lower rate of progression may be found in populations including patients with all the different patterns of hip osteoarthritis.

Although we focused on a particular subset of patients, our study enabled us to identify some variables that might influence the radiological progression of hip osteoarthritis, confirming results from previous clinical epidemiological studies using different methodologies that demonstrated the importance of femoral head migration, ${ }^{5817}$ gender, ${ }^{17}{ }^{18}$ and age. ${ }^{58} \mathrm{We}$ also found that clinical activity at entry, evaluated by Lequesne's functional index, might be predictive of radiological progression. The level of functional disability is difficult to ascertain and difficult to interpret, but it may be argued that part of this clinical functional disability might be explained by a 'flare' of the disease (an inflammatory process), and that this inflammatory process might lead to an episode of chondrolysis. ${ }^{24}$ The influence of obesity and polyarticular involvement on the anatomical progression remains unclear, with conflicting results among studies; ${ }^{25-32}$ these variables were not identified in our study.

The demonstration of a correlation between clinical symptoms and radiographic features is not easy in osteoarthritis: factors differentiating symptomatic osteoarthritis from asymptomatic disease are unknown, and the present study emphasises that a cross-sectional design is not appropriate to evaluate such a correlation. In contrast, a longitudinal study in which the correlations between changes in radiological parameters and changes in the clinical parameters were evaluated achieved statistical significance $(p<0.0001)$. Even this correlation seems moderate, however, as all the clinical parameters explained only $15 \%$ of the variability of the radiological change in joint space width. More intriguing are the possible correlations between drug intake and radiological progression of hip osteoarthritis. Taking into account clinical parameters (level of pain and functional disability), we failed to demonstrate a statistically significant correlation between analgesic consumption score and radiological progression, but found a statistically significant correlation between NSAID consumption score and radiological progression. These results raise the question of a deleterious effect of NSAID consumption on cartilage breakdown, as has previously been suggested 
to occur after long term intake of indomethacin. ${ }^{33}$ Our study does not permit differentiation between the effects of the various NSAIDs, but strongly emphasises the need to pursue clinical research in this field.

This study was supported in part by a grant from Negma Ltd.

1 Tepper S, Hochberg M C. Factors associated with hip osteoarthritis: data from the first National Health and osteoarthritis: data from the first National Health and Epidemiol 1993; 137: 1081-8.

2 Felson D T. Epidemiology of hip and knee osteoarthritis. Epidemiol Rev 1988; 10: 1-18.

3 Lawrence R C, Hochberg M C, Kelsey J L, et al. Estimates of the prevalence of selected arthritic and musculoskeletal diseases in the United States. F Rheumatol 1989; 16: $427-41$.

4 Hoaaglund F T, Yau S C M C, Wong W L. Osteoarthritis of the hip and other joints in southem Chinese in Hon Kong. $\mathcal{F}$ Bone foint Surg Am 1973; 55: 545-57.

5 Ledingham J, Dawson S, Preston B, Milligan G Doherty M. Radiographic progression of hospital referred osteoarthritis of the hip. Ann Rheum Dis 1993; 52: 263-7.

6 Seifert M H, Whiteside C G, Savage O. A 5-year follow-up of fifty cases of idiopathic osteoarthritis of the hip. Ann of fifty cases of idiopathic oste

7 Macys J R, Bullough P G, Wilson P D Jr. Coxarthrosis: a study of the natural history based on a correlation of clinical, radiographic, and pathologic findings. Semin Arthritis Rheum 1980; 10: 66-80.

8 Danielsson L. Incidence and prognosis of coxarthrosis. Acto Orthop Scand 1964; 66 (suppl): 9-87.

9 Dieppe P, Cushnaghan J. Prospective study of outcome in osteoarthritis. Arthritis Rheum 1988; 31 (suppl 4): S74.

10 Ravaud P, Auleley G R, Amor B, Dougados M. Radiographic assessment of progression in knee osteoarthritis Rheumatol Europe 1995; 24 (suppl 2): 129-31.

11 Kellgren J H, Lawrence J S. Radiologic assessment of osteoarthritis. Ann Rheum Dis 1957; 16: 494-502.

12 Lequesne $M$. Quantitative measurements of joint space during progression of osteoarthritis: "Chondrometry". In: during progression of osteoarthritis: "Chondrometry". In
K E Huettner, V M Goldberg, eds. Osteoarthritic disorders. Rosemont: American Academy of Orthopaedic Surgeons, $1994 ; 427-44$

13 Dougados M, Villers C, Amor B. Sensitivity to change of various roentgenological severity scoring systems for osteoarthritis of the hip. Rev Rhum [English edn] 1995; 62: 169-73.

14 Conrozier T, Ron A M, Balblanc J C, et al. Measurement of the hip joint space using computerized image analysis. Rev Rhum [English edn] 1993; 60: 105-11.

15 Conrozier T, Tron A M, Mathieu P, Vignon E. Quantitative assessment of radiographic normal and osteoarthritic hip joint space. Osteoarthritis Cartilage 1995; 3 (suppl A): joint $81-8$.
16 Dieppe P, Cushnaghan J, Jasani M K, et al. A two-year placebo-controlled trial of non-steroidal anti-inflammatory therapy in osteoarthritis of the knee joint. $\mathrm{Br} F$ Rheumatol 1993; 32: 595-600.

17 Jorring K. Osteoarthritis of the hip. Epidemiology and clinical role. Acta Orthop Scand 1980; 51: 523-30.

18 Katz J N, Wright E A, Guadagnoli E, Liang $H$, Karlson E W, Cleary P D. Differences betwen men and women undergoing major orthopedic surgery for degenerative arthritis. Arthritis Rheum 1994; 37: 687-94.

19 Goldberg R L, Huff J P, Lenz M E, Glickman P, Katz R, Thonar E J M. Elevated plasma levels of hyaluronate in patients with osteoarthritis and rheumatoid arthritis. Arthritis Rheum 1991; 34: 799-806.

20 Lequesne M, Mery C, Samson M, Gerard P. Indexes of severity for osteoarthritis of the hip and knee. Scand $\mathcal{F}$ Rheumatol 1987; 65: 85-9.

21 Dougados M, Nguyen M, Listrat V, Amor B. Score d'equivalence des anti-inflammatoires non stéroïdiens (A.I.N.S.). Rev Rhum Mal Osteoarctic 1989; 56: 251 [abstract 1134].

22 Bland J M, Altman D G. Statistical methods for assessing agreement between two methods of clinical assessment. Lancet 1986; i: $307-10$.

23 Conrozier $T$, Lequesne $M$, Tron A $M$, Mathieu $P$, Berdah L, Vignon $E$. The effects of position on the radiographic joint space in osteoarthritis of the hip. Osteoarthritis Cartilage 1996. In press.

24 Amor B Congestive outbreak of osteoarthritis: chondrolysis and cartilage repair. Revue du Praticien 1993; 43: 601-3.

25 Kraus J F, D'Ambrosia R D, Smith E G, et al. An epidemiological study of severe osteoarthritis. Orthopedics 1978; 1:37-42.

26 Hartz A J, Fischer M E, Bril G, et al. The association of obesity with joint pain and osteoarthritis in the HANES data. $₹$ Chron Dis 1986; 39: 311-9.

27 Vingard E. Overweight predisposes to coxarthrosis. Bodymass index studied in 239 males with hip arthroplasty. Acta Orthop Scand 1991; 62: 106-9.

28 Rissanen A, Heliovaara M, Knekt P, Reunanen A, Aromaa A, Maatela J. Risk of disability and mortality due to overweight in a Finnish population. BMF 1990; 301: 835-7.

29 Van Saase J L C M, Vanderbroucke J P, Van Romunde L KJ, Valkenburg H A. Osteoarthritis and obesity in the general Valkenburg H A. Osteoarthritis and obesity in the general population. A relationship call

30 Saville P D, Dickson J. Age and weight in osteoarthrosis of the hip. Arthritis Rheum 1968; 11: 635-44.

31 Dougados $M$, Gueguen A, Nguyen $M$, et al. Longitudinal radiologic evaluation of osteoarthritis of the knee. $f$ Rheumatol 1992; 19: 378-84.

32 Perry G H, Smith M J G, Whiteside C G. Spontaneous recovery of the joint space in degenerative hip disease. Ann Rheum Dis 1972; 31: 440-8.

33 Dacre J, Byrne J, Huskisson E C, and the LINK study group. Use of a simple scoring system for knee osteoarthritis to compare indomethacin, placebo and tiaprofenic and a long-term double blind controlled trial in osteoarthritis. Arthritis Rheum 1994; 27 (suppl 9): [abstract 1566]. 